\title{
Automedicação em pacientes renais crônicos hemodialíticos
}

\section{Self-medication in chronic kidney disease patients on hemodialysis Automedicación de pacientes renales crónicos en hemodiálisis}

\author{
Lucas Brasileiro Lemos (iD \\ Universidade Estadual do Sudoeste da Bahia - UESB - Jequié (BA) - Brasil \\ Gabriela Silva Moraes iD \\ Universidade Estadual do Sudoeste da Bahia - UESB - Jequié (BA) - Brasil \\ Gisele da Silveira Lemos \\ Universidade Estadual do Sudoeste da Bahia - UESB - Jequié (BA) - Brasil \\ Adriana Alves Nery \\ Universidade Estadual do Sudoeste da Bahia - UESB - Jequié (BA) - Brasil
}

\section{RESUMO}

Objetivo: Analisar o uso de medicamentos por automedicação em pacientes renais crônicos hemodialíticos. Métodos: Estudo quantitativo, descritivo e transversal, realizado em um centro de tratamento hemodialítico, na região Sudoeste da Bahia, em março de 2015, por meio da aplicação de formulário contendo variáveis sociodemográficas, clínicas e farmacoterapêuticas de 170 pacientes. A amostra compôs-se de pacientes em tratamento hemodialítico crônico há mais de um ano, com idade maior ou igual a 18 anos. Utilizou-se o Epidata 3.1 como banco de dados. Realizou-se análise descritiva e empregou-se o método de regressão logística binária, usado para avaliar a associação entre automedicação e variáveis independentes, utilizando o programa SPSS, versão 21.0. Resultados: Dos participantes, 64,1\% (109) eram homens, com idade média de 50,5 anos ( \pm 14,9); $57 \%$ (98) possuíam renda menor que um salário mínimo; $20 \%$ (34) nunca estudaram; $48,2 \%$ (82) estavam dialisando em período de um a cinco anos e 92,9\% (158) não possuíam plano de saúde. Contabilizaram-se 104 medicamentos utilizados por automedicação, com destaque para o calcitriol $(9,6 \%)$ e a clonidina $(6,7 \%)$. Verificou-se que pacientes com maior escolaridade $(\mathrm{OR}=1,32$; IC95\%=1,32-28,72) e que usam sobra de medicamentos em casa $(\mathrm{OR}=22,48 ; \mathrm{IC} 95 \%=6,53-77,38)$ têm mais chances de se automedicar. Conclusão: Há baixa frequência de automedicação na população de renais crônicos investigada, sendo associada ao uso de medicamentos guardados em casa e à baixa escolaridade.

Descritores: Diálise Renal; Farmacoepidemiologia; Medicamentos Sem Prescrição; Uso de Medicamentos; Pacientes Ambulatoriais; Insuficiência Renal Crônica.

\section{ABSTRACT}

Objective: To analyze self-medication in chronic kidney disease patients on hemodialysis. Methods: A quantitative descriptive cross-sectional study was conducted at a hemodialysis center in Southwestern Bahia in March 2015 using a form to collect sociodemographic, clinical and drug therapy data from 170 patients in March 2015. The sample comprised patients undergoing chronic hemodialysis treatment for over one year aged 18 years or older. Epidata 3.1 was used as a database. Descriptive analysis was performed and binary logistic regression was used to check for associations between self-medication and independent variables using the SPSS software version 21.0. Results: In all, 64.1\% (109) of the participants were men and the mean age was 50.5 years ( \pm 14.9$) .57 \%$ (98) of the participants earned less than one minimum wage, $20 \%$ (34) had never studied, $48.2 \%$ (82) had been on dialysis for one to five years, and $92.9 \%$ (158) had no health insurance. We confirmed self-medication of 104 drugs, particularly calcitriol (9.6\%) and clonidine (6.7\%). Patients with higher levels of education (OR=1.32; 95\% Cl=1.32-28.72) and those who use leftover drugs at home $(\mathrm{OR}=22.48 ; 95 \% \mathrm{Cl}=6.53-77.38)$ were more likely to self-medicate. Conclusion: The rate of self-medication in chronic kidney disease patients is low and it is associated with the use of drugs stored at home and low levels of education.

Descriptors: Renal Dialysis; Pharmacoepidemiology; Nonprescription Drugs; Drug Utilization; Outpatients; Renal Insufficiency, Chronic.

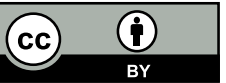




\section{RESUMEN}

Objetivo: Analizar el uso de medicamentos por automedicación de pacientes renales crónicos en hemodiálisis. Métodos: Estudio cuantitativo, descriptivo y transversal realizado en un centro de tratamiento de hemodiálisis de la región Sudoeste de Bahía en marzo de 2015 a través de la aplicación de un formulario con variables sociodemográficas, clínicas y de tratamiento farmacológico de 170 pacientes. La muestra ha sido de pacientes en tratamiento de hemodiálisis desde hace más de un año con edad mayor o igual a 18 años. Se utilizó el Epidata 3.1 para el banco de datos. Se realizó un análisis descriptivo y se utilizó el método de regresión logística binaria para evaluar la asociación entre la automedicación y las variables independientes con el programa SPSS, versión 21.0. Resultados: Entre los participantes, el 64,1\% (109) era hombres con edad media de 50,5 años ( $\pm 14,9)$; el $57 \%$ (98) tenía renta de menos de un sueldo mínimo; el 20\% (34) nunca ha estudiado; el 48,2\% (82) realizaba la hemodiálisis desde el periodo entre uno y cinco años y el 92,9\% (158) no tenía seguro de salud. Se ha contabilizado 104 medicamentos utilizados por la automedicación con énfasis para el calcitriol (9,6\%) y la clonidina (6,7\%). Se verificó que los pacientes con mayor escolaridad (OR=1,32; IC95\%=1,32-28,72) y los que usan lo que queda de los medicamentos que tienen en casa (OR=22,48; IC95\%=6,53-77,38) tienen más oportunidades para la automedicación. Conclusión: Hay baja frecuencia de automedicación en la población de renales crónicos investigados y la misma se asoció con el uso de medicamentos almacenados en casa y las personas de baja escolaridad.

Descriptores: Diálisis Renal; Farmacoepidemiología; Medicamentos sin Prescripción; Utilización de Medicamentos; Pacientes Ambulatorios; Insuficiencia Renal Crónica.

\section{INTRODUÇÃO}

A doença renal crônica (DRC) encontra-se em epidemia no mundo, com uma importante e representativa carga social e econômica para a população no contexto de saúde pública ${ }^{(1)}$. A hipertensão arterial sistêmica (HAS) e o diabetes mellitus (DM) se destacam como as principais causas primárias da DRC, 34 e $31 \%$ respectivamente ${ }^{(2)}$. $O$ conhecimento dos aspectos epidemiológicos e clínicos dos pacientes dialíticos funciona como subsídio aos gestores no planejamento de uma assistência segura e promoção da qualidade vida, com redução da morbimortalidade ${ }^{(2-4)}$.

Em 2016, nos Estados Unidos, 124.675 pacientes novos haviam iniciado o tratamento dialítico e estimou-se um custo de cerca de 3,7 bilhões de dólares referente a medicamentos para os pacientes com doença renal crônica terminal (DRCt) inscritos no programa Medicare Part $D$, incluindo os pacientes em diálise e transplantados ${ }^{(5)}$. No Brasil, 40.307 novos pacientes iniciaram tratamento dialítico em 2017, ou seja, incidência estimada de 194 pacientes por milhão de habitantes (PMP). A taxa de prevalência estimada de pacientes em diálise crônica aumentou globalmente de 475 PMP, em 2011, para 610 PMP, em $2017(28,4 \%)^{(2)}$. Dessa forma, evidencia-se que a melhor opção para trabalhar a promoção da saúde dessa população é a prevenção pelo diagnóstico precoce de DRC, em um dos seus cinco estágios, e preservação da função renal|(3).

Alerta-se que os pacientes com DRCt têm indicadores de mortalidade e morbidade mais elevados em relação à população geral( ${ }^{(6)}$, com taxa de mortalidade anual de 19,9\%, no Brasil, em $2017^{(2)}$. Esses pacientes costumam necessitar de um número elevado de medicamentos, em decorrência do crescente envelhecimento em devido à diálise e de suas características relacionadas ao seu estado inflamatório crônico, do hiperparatireoidismo, da anemia, da má nutrição e da presença de comorbidades múltiplas, como diabetes, dislipidemia e hipertensão arterial ${ }^{(6)}$. Acrescenta-se que há uma maior probabilidade de ocorrência de eventos adversos nesses pacientes, fazendo com que seja revista a real necessidade do uso de diversos medicamentos ${ }^{(7)}$.

O conhecimento do perfil farmacoterapêutico dos pacientes torna-se uma ferramenta de grande utilidade na identificação de problemas relacionados aos medicamentos (PRM) e um de seus fatores de risco: a automedicação( ${ }^{(8)}$, que se define como a seleção e uso de medicamentos por conta própria, para tratar doenças ou sintomas autorreconhecidos, incluindo a aquisição de drogas sem prescrição, o uso de doses de sobras de prescrições antigas e de drogas compartilhadas com outros membros da família ou grupo social, e o uso inadequado de prescrição médica por prolongá-la, interrompê-la ou modificar a dosagem e o período da administração( ${ }^{(9)}$.

Encontram-se, entre os riscos relacionados com a automedicação, o perigo da polifarmácia e de várias interações medicamentosas, como também a chance de superdosagem e intoxicação, podendo ser intensificados na população de renais crônicos pelas suas características peculiares em relação à farmacocinética ${ }^{(10)}$. Dessa forma, estudos sobre a utilização de medicamentos (EUM) nessa população fornecem dados importantes aos gestores para a confecção de políticas públicas de saúde ${ }^{(11)}$, uma vez que doenças ou condições crônicas mais graves podem levar ao uso de medicamentos prescritos ${ }^{(12)} \mathrm{e}$ a presença dessas doenças crônicas pode ser um dos fatores determinantes para a 
prática da automedicação( ${ }^{(13)}$. Diante desses dados, objetiva-se, no presente estudo, analisar o uso de medicamentos por automedicação em pacientes renais crônicos hemodialíticos.

\section{MÉTODOS}

Trata-se de um estudo quantitativo, descritivo e transversal, realizado em um centro de tratamento hemodialítico, na região Sudoeste da Bahia, Brasil, em março de 2015. Do total de 250 pacientes do centro, selecionaram-se 189 por atenderem aos seguintes critérios de inclusão: pacientes hemodialíticos crônicos, com tempo de tratamento superior a um ano ${ }^{(14)}$ e idade maior ou igual a 18 anos. Desses pré-selecionados, apenas 170 responderam ao formulário aplicado. A perda de $10 \%$ da amostra se deu por motivos como recusa, óbito, transferências de serviços ou mudança de modalidade de tratamento.

Realizou-se estudo piloto para a adequação do instrumento de coleta de dados e calibração dos entrevistadores com pacientes do mesmo centro de tratamento inclusos na amostra selecionada, uma vez que não houve necessidade de mudanças na estrutura do formulário, construído por uma equipe multidisciplinar de saúde. Após assinatura do Termo de Consentimento Livre e Esclarecido pelos participantes, esses responderam ao formulário da pesquisa.

Compuseram o instrumento de coleta perguntas referentes a dados sociodemográficos (sexo, idade, raça, escolaridade, renda familiar mensal, religião, estado civil, ocupação, coabitação), além de outras questões específicas relacionadas à saúde do participante e aos serviços de saúde, tais como: a data da última consulta médica, a presença de comorbidades (como DM e HAS) concomitantes à doença renal, hábitos de vida (independente do tratamento hemodialítico) e dados sobre medicamentos, como nome dos medicamentos que faziam uso, origem da recomendação do uso (prescrição médica ou de outros profissionais), número de vezes em que se automedicou e características associadas à prática de se automedicar. Considerou-se, sobre a data da última consulta com algum médico, apenas aquela em que o paciente foi atendido em consultório médico.

Empregou-se, para o termo automedicação, quando envolveu alguma variável temporal na questão, um período recordatório de 30 dias, semelhante a um estudo realizado em Pereira, na Colômbia ${ }^{(15)}$.

Acrescenta-se, quanto aos medicamentos consumidos, que foram levados em consideração se apresentadas as bulas, embalagens ou prescrições, semelhante a um estudo realizado no Distrito Federal ${ }^{(16)}$, mas se incluiu também o autorrelato. Definiram-se como medicamentos prescritos aqueles constantes em receituários médicos e, quando não eram apresentados, mas o paciente relatava o uso ou trazia bulas ou embalagens, se tais medicamentos estivessem prescritos no prontuário eletrônico do paciente, sendo feita uma posterior conferência pelo pesquisador. Assim, quando não enquadrados nesses critérios, classificaram-se os medicamentos como não prescritos ou como automedicação, mesmo que o prolongamento do uso de uma droga previamente prescrita pudesse ter ocorrido por uma suposta falha na comunicação entre a equipe médica e os pacientes.

Classificaram-se os medicamentos também como de uso na clínica ou no domicílio. Dúvidas, inconsistências e itens do formulário não preenchidos durante a primeira abordagem eram complementados em uma segunda abordagem do participante, tendo sido ambas previamente agendadas. Os pacientes e/ou acompanhantes recebiam, durante a sessão de diálise anterior à do dia da entrevista, um lembrete impresso contendo orientações, que eram cuidadosamente explicadas.

Classificaram-se as medicações em uso e seus respectivos princípios ativos em cada especialidade de acordo com o sistema de classificação Anatômica Terapêutico Química (Anatomical Therapeutic Chemical - ATC) da Organização Mundial de Saúde ${ }^{(17)}$.

Utilizou-se o programa Epidata, versão 3.1, para a tabulação dos dados coletados e o Statistical Package for the Social Sciences (SPSS), versão 21.0, para o processamento e a análise dos dados. Apresentaram-se as análises descritivas por meio de frequências (absoluta e relativa), médias e desvio-padrão. Realizou-se a associação, uni e multivariada, entre a automedicação e as variáveis independentes selecionadas empregando-se o modelo de regressão logística binária. Calculou-se a magnitude da associação por meio de odds ratios (OR), com intervalos de confiança de $95 \%$ e nível de significância de 0,05.

Verificou-se o efeito independente das variáveis sobre a automedicação pela análise multivariada, e realizou-se a estratégia de construção dos modelos pelo processo de deleção de variáveis até a obtenção do modelo final, em que permaneceram aquelas com $p<0,05$. Fez-se a adequação do ajuste pelo teste de Hosmer-Lemeshow.

Primou-se pelo atendimento às questões éticas que envolvem pesquisas com seres humanos, conforme o previsto na Resolução n. ${ }^{\circ}$ 466/12 do Conselho Nacional de Saúde/Ministério da Saúde. Obteve-se autorização para o estudo do Comitê de Ética em Pesquisa (CEP) da Universidade Estadual do Sudoeste da Bahia, conforme Parecer n. ${ }^{\circ} 957.553$. 


\section{RESULTADOS}

Dos 170 participantes do presente estudo, $74(43,5 \%)$ se declararam como pardos e $54(31,8 \%)$ negros. Na situação conjugal, 62 (36,5\%) são casados e 31 (18,2\%) amasiados. Dos participantes alfabetizados, 97 (71,3\%) obtiveram aprovação no último ano do ensino fundamental. Além disso, $164(96,5 \%)$ são residentes na área urbana e $144(84,7 \%)$ residiam em companhia de seus familiares. A idade variou entre 20 e 86 anos, com média de 50,5 anos $( \pm 14,9)$, e o tempo médio de tratamento hemodialítico perpassa 61,5 meses $( \pm 61,3)$, como consta na Tabela I.

Tabela I - Características da população do estudo, segundo variáveis sociodemográficas, clínicas e do acesso a serviços de saúde. Jequié, Bahia, Brasil, 2015.

\begin{tabular}{|c|c|c|}
\hline Variáveis & $\mathbf{n}$ & $\%$ \\
\hline \multicolumn{3}{|l|}{ Sexo } \\
\hline Masculino & 109 & 64,1 \\
\hline Feminino & 61 & 35,9 \\
\hline \multicolumn{3}{|l|}{ Situação conjugal } \\
\hline Solteiro & 56 & 32,9 \\
\hline Outros* & 114 & 67,1 \\
\hline \multicolumn{3}{|l|}{ Raça/ Cor } \\
\hline Branco & 40 & 23,5 \\
\hline Não branco"* & 130 & 76,5 \\
\hline \multicolumn{3}{|l|}{ Com quem vive } \\
\hline Familiar & 144 & 84,7 \\
\hline Sozinho+ outros & 26 & 15,3 \\
\hline \multicolumn{3}{|l|}{ Renda familiar mensal } \\
\hline Até 1 salário & 98 & 57,6 \\
\hline Até 2 salários ou acima & 72 & 42,4 \\
\hline \multicolumn{3}{|l|}{ Escolaridade } \\
\hline Não alfabetizado & 34 & 20,0 \\
\hline Alfabetizado"** & 136 & 80,0 \\
\hline \multicolumn{3}{|l|}{ Possui plano de saúde } \\
\hline Sim & 12 & 7,1 \\
\hline Não & 158 & 92,9 \\
\hline \multicolumn{3}{|l|}{ Saúde atual } \\
\hline Muito boa e boa & 73 & 42,9 \\
\hline Regular & 78 & 45,9 \\
\hline Muito ruim e ruim & 19 & 11,2 \\
\hline \multicolumn{3}{|l|}{ Tempo em hemodiálise } \\
\hline 1 a 5 anos & 82 & 48,2 \\
\hline Acima de 5 anos & 88 & 51,8 \\
\hline \multicolumn{3}{|l|}{ DM elou HAS } \\
\hline Sim & 136 & 80,0 \\
\hline Não & 34 & 20,0 \\
\hline \multicolumn{3}{|l|}{ Última consulta } \\
\hline Até um mês & 41 & 24,1 \\
\hline Acima de um mês & 129 & 75,9 \\
\hline \multicolumn{3}{|l|}{ Limitação para locomover } \\
\hline Sim & 29 & 17,1 \\
\hline Não & 141 & 82,9 \\
\hline
\end{tabular}

"Outros: casado, divorciado, amasiado, viúvo; "Não branco: pardo, negro e amarelo; '”Alfabetizado: ensino fundamental, ensino médio e ensino superior; DM: diabetes mellitus; HAS: hipertensão arterial sistêmica 
Notou-se, dos participantes que disseram ter usado medicamento sem orientação ou receita médica, que apenas 3 participantes $(1,8 \%)$ se automedicaram por medo de ir ao médico. Houve $30(17,6 \%)$ pacientes que relataram já ter usado medicamentos guardados em casa, sendo 19 (63,3\%) por meio de sobras de receitas anteriores. Em relação às atitudes consideradas como automedicação, aqueles participantes que deixaram, por conta própria, de tomar algum medicamento prescrito nos últimos 30 dias, descreveram os seguintes motivos: preço ( $n=2 ; 14,3 \%)$, medicamento em falta ( $n=3 ; 21,4 \%)$, não tinha transporte para buscar $(n=1 ; 7,1 \%)$, não gosta de tomar o medicamento $(n=2 ; 14,3 \%)$, não se sente bem quando toma o medicamento $(n=6 ; 42,9 \%)$ e, ainda, $53(75 \%)$ participantes que usaram medicamento por conta própria relataram que sabiam como usá-lo e que melhoraram após o uso, de acordo a Tabela II.

Diante do relato de ter se automedicado em algum momento de sua vida, apenas 2 participantes $(3 \%)$ relataram ter sido influenciados por propagandas da mídia e $13(19,4 \%)$, pelo preço do medicamento. Apesar de 152 pacientes reconhecerem que tinham sido orientados a evitar a prática de automedicação, ainda assim, $11(6,4 \%)$ afirmaram ter usado medicamento por conta própria durante a sessão de hemodiálise, conforme apresenta a Tabela II.

Tabela II - Características da prática de automedicação dos pacientes renais crônicos hemodialíticos. Jequié, Bahia, Brasil, 2015.

\begin{tabular}{|c|c|c|c|}
\hline \multirow[b]{2}{*}{ Características de automedicação } & \multicolumn{3}{|c|}{ Práticas de automedicação } \\
\hline & n (\%) & n (\%) & n (\%) \\
\hline Sabe o que é automedicação? & $\underset{58(34,1)}{\operatorname{Sim}}$ & $\begin{array}{c}\text { Não/ Talvez } \\
112(65,9)\end{array}$ & - \\
\hline Usou medicamento sem orientação ou receita médica? & $\begin{array}{l}\operatorname{Sim} \\
67(39,4)\end{array}$ & $\begin{array}{c}\text { Não/ Não lembro } \\
103(60,6)\end{array}$ & - \\
\hline Se resposta negativa, DRC influenciou em evitar? & $\underset{\text { Sim }}{73(70,9)}$ & $\begin{array}{c}\text { Não } \\
30(29,1)\end{array}$ & - \\
\hline Se a resposta foi positiva, lembra quando usou? & $\begin{array}{l}>30 \text { dias } \\
29(43,3)\end{array}$ & $\begin{array}{l}<30 \text { dias } \\
14(20,9)\end{array}$ & $\begin{array}{c}\text { Não recorda } \\
24(35,8)\end{array}$ \\
\hline Quem recomendou o uso? & $\begin{array}{l}\text { Conta própria } \\
59(88,0)\end{array}$ & $\begin{array}{l}\text { Outros* } \\
8(12,0)\end{array}$ & - \\
\hline Deixou de tomar medicamento prescrito nos últimos 30 dias? & $\underset{14(8,2)}{\operatorname{Sim}}$ & $\begin{array}{c}\text { Não } \\
156(91,8)\end{array}$ & - \\
\hline Aumentou dose por algum motivo? & $\begin{array}{c}\text { Não melhorou } \\
1(0,6)\end{array}$ & $\begin{array}{c}\text { Nunca aumentou } \\
169(99,4)\end{array}$ & - \\
\hline Sinais ou sintomas que o levou a se automedicar? & $\begin{array}{l}\text { Dor de cabeça } \\
\quad 38(56,7)\end{array}$ & $\begin{array}{l}\text { Outros }^{* *} \\
29(43,3)\end{array}$ & - \\
\hline Como conseguiu o medicamento? & $\begin{array}{l}\text { Sozinho } \\
61(91,0)\end{array}$ & $\begin{array}{c}\text { Ajuda de outros } \\
6(9,0)\end{array}$ & - \\
\hline Medicamentos necessitaram de receita obrigatória? & $\begin{array}{c}\text { Não } \\
70(98,6)\end{array}$ & $\begin{array}{c}\text { Não sei } \\
1(1,4)\end{array}$ & - \\
\hline Como a saúde ficou após o uso? & $\begin{array}{l}\text { Melhorou } \\
53(74,6)\end{array}$ & $\begin{array}{c}\text { Piorou/ não mudou } \\
18(25,4)\end{array}$ & - \\
\hline
\end{tabular}

*Outros: familiar, balconista de farmácia, farmacêutico, vizinho; * Outros: tosse, cansaço/fadiga e outros; DRC: doença renal crônica

Entre os participantes que se automedicaram nos últimos trinta dias $(n=51 ; 30 \%)$, observou-se o uso de 103 medicamentos, média de 2,04 $( \pm 1,40)$. Detalha-se que, apesar de o principal motivo para o uso da automedicação ter sido a cefaleia, medicamentos com propriedades analgésicas, como a nimesulida e a dipirona, encontram-se como o terceiro e quarto medicamentos de maior frequência de uso entre os classificados como não prescritos ou por automedicação, atrás do calcitriol e da clonidina, como dispõe a Tabela III. 
Tabela III - Distribuição dos medicamentos classificados como não prescritos, segundo classificação anatômica terapêutica. Jequié, Bahia, Brasil, 2015.

\begin{tabular}{|c|c|c|}
\hline Não prescritos por subgrupo terapêutico (ATC) & $\mathbf{n}$ & $\%$ \\
\hline \multicolumn{3}{|l|}{ A- Sistema digestivo e metabolismo } \\
\hline Calcitriol & 10 & 9,7 \\
\hline Outros & 20 & 19,4 \\
\hline \multicolumn{3}{|l|}{ B- Sangue e órgãos hematopoiéticos } \\
\hline Hidróxido de ferro & 3 & 2,9 \\
\hline Outros & 4 & 3,9 \\
\hline \multicolumn{3}{|l|}{ C- Sistema cardiovascular } \\
\hline Clonidina & 7 & 6,8 \\
\hline Outros & 19 & 18,4 \\
\hline \multicolumn{3}{|l|}{ M- Sistema musculoesquelético } \\
\hline Nimesulida & 6 & 5,8 \\
\hline Outros & 11 & 10,7 \\
\hline \multicolumn{3}{|l|}{$\mathrm{N}$ - Sistema nervoso } \\
\hline Dipirona & 6 & 5,8 \\
\hline Outros & 4 & 3,9 \\
\hline \multicolumn{3}{|l|}{ R-Sistema respiratório } \\
\hline Dropropizina, expector mel ${ }^{\circledR}$, resfryneo ${ }^{\circledR}$ & 3 & 2,9 \\
\hline \multicolumn{3}{|l|}{ V- Vários } \\
\hline Cloridrato de sevelâmer & 6 & 5,8 \\
\hline Outros & 1 & 1,0 \\
\hline Outros* & 3 & 3,0 \\
\hline Total & 103 & 100 \\
\hline
\end{tabular}

®: marca registrada; Cl: cloridrato; Outros: J - anti-infecciosos, L- agentes antineoplásicos, H- hormônios de uso sistêmico; ATC: Anatômica Terapêutico Química

Relacionaram-se à automedicação, na análise univariada, a escolaridade, o uso de medicamentos guardados em casa e a identificação do medicamento pelo nome. Já as variáveis escolaridade e uso de medicamentos guardados em casa estiveram independentemente associadas à prática de automedicação, como expõe a Tabela IV.

Tabela IV - Resultado da análise multivariada para comparação de variáveis selecionadas e automedicação. Jequié, Bahia, Brasil, 2015.

\begin{tabular}{lcccc}
\hline \multirow{2}{*}{ Variáveis } & \multicolumn{2}{c}{ Automedicação } & \multicolumn{2}{c}{ Análise multivariada } \\
\cline { 2 - 5 } & Sim $\mathbf{n}(\%)$ & Não $\mathbf{n}(\%)$ & OR/ IC & p-valor* \\
\hline Usou medicamentos guardados em casa & & & & \\
$\quad$ Não & $41(29,3)$ & $99(70,7)$ & $22,48(6,53-77,38)$ & $<0,01$ \\
$\quad$ Sim & $26(86,7)$ & $4(13,3)$ & 1 & \\
Escolaridade & & & & \\
$\quad$ Não alfabetizado & $4(11,8)$ & $30(88,2)$ & $6,15(1,32-28,72)$ & 0,021 \\
$\quad$ Alfabetizado & $63(46,3)$ & $73(53,7)$ & 1 & \\
Teste de Hosmer-Lemeshow & & & \multicolumn{2}{c}{0,596} \\
\hline
\end{tabular}

Med: medicamento; "Regressão logística binária, $p<0,05$ 


\section{DISCUSSÃO}

Entende-se que estudos como este, envolvendo o tema automedicação, muitas vezes costumam ter resultados divergentes em função de diferentes abordagens metodológicas e períodos recordatórios utilizados, fato que também é tratado em um estudo realizado no Distrito Federal ${ }^{(16)}$. Apesar das dificuldades, esses estudos são importantes como estratégias para a promoção da saúde, evitando problemas relacionados a medicamentos e objetivando a melhora da qualidade de vida dos pacientes ${ }^{(2-4,11)}$.

A frequência de automedicação encontrada no presente estudo aproxima-se a outros, como a encontrada em um estudo com adultos na Colômbia ${ }^{(15)}$ e entre estudantes da Palestina ${ }^{(18)}$, ambos com período recordatório de 30 dias. Encontrou-se, em estudo realizado em Campinas com um grupo de homens, um valor de $11,2 \%$, sendo usado um recordatório de apenas três dias ${ }^{(12)}$.

Observou-se, quando não determinado o período recordatório, uma prevalência de $53,2 \%$ em um estudo realizado na Nigéria entre estudantes de Farmácia ${ }^{(19)}$. Podem parcialmente designar diferenças na prevalência do uso de determinados medicamentos e de automedicação as características das populações, seu estado de saúde, a utilização de serviços e modelo de atenção à saúde, assim como as características sociodemográficas e culturais ligadas à utilização de medicamento ${ }^{(12)}$, se fazendo necessário o conhecimento sobre essa prática para o direcionamento de políticas públicas a essa população.

Contabilizou-se na presente pesquisa um total de 104 medicamentos não prescritos utilizados, uma média de $2,04( \pm 1,4)$, sendo que, em um trabalho realizado entre estudantes universitários na Palestina ${ }^{(18)}$, encontrou-se um número médio de 2,63 $( \pm 1,38)$. A baixa frequência de automedicação, somada com a baixa média de medicamentos utilizados, reforça o relato dos pacientes que negaram se automedicar considerando o fato de possuírem DRC, colaborando com dados da literatura, que citam que a frequência de automedicação costuma ser menor em pacientes com doenças crônicas ${ }^{(11,20)}$.

Demonstra-se, particularmente em relação à população estudada, que os doentes renais crônicos hemodialíticos têm acesso ao serviço de saúde três vezes por semana, no mínimo, e, além disso, o fato de comorbidades como DM e HAS serem prevalentes os fazem buscar por medicamentos e exames com maior periodicidade, o que os deixa em maior contato com o profissional médico, favorecendo o uso de medicamentos prescritos ${ }^{(12)}$. Há relato na literatura, no entanto, de associação positiva de doença crônica com a automedicação( ${ }^{(13)}$.

Observou-se, em um estudo realizado com universitários do estado de Santa Catarina, a controversa associação da automedicação com acesso a serviços de saúde, sendo ela, às vezes, mais frequente entre aqueles que usavam os serviços de saúde. Inclusive, chegando a ser referida como se fosse um indicador de substituição da atenção formal à saúde, de autocuidado, ajudando na recuperação de pequenas indisposições e podendo aliviar um pouco a sobrecarga dos serviços médicos ${ }^{(21)}$.

Revela-se, na atual pesquisa, que entre os medicamentos mais encontrados como de uso sem prescrição, os mais frequentes foram o calcitriol e a clonidina. Torna-se possível que tenha ocorrido algum erro de concordância medicamentosa, principalmente em relação ao primeiro, entre o que era visto na prescrição eletrônica e o que o paciente relatava estar usando. Nesse caso, possivelmente o medicamento tenha sido suspenso recentemente da prescrição mensal mais atual e o paciente ainda não tivesse recebido a receita. A suposta falha na comunicação entre prescritores e usuários, em caso de haver suspensão do medicamento pelo médico e o paciente não ser comunicado ou não compreender o tratamento, é considerada um problema de segurança do paciente ${ }^{(22)}$. Pontuase que os problemas de comunicação nos estabelecimentos de saúde estão entre os mais encontrados em análise de causa raiz dos eventos sentinela ${ }^{(22)}$, por isso os profissionais devem se preocupar em confirmar que o paciente compreendeu o tratamento proposto e/ ou sua alteração, mitigando os riscos relacionados com a automedicação(8) e promovendo uma melhora na qualidade de vida ${ }^{(3)}$.

Averiguou-se que existem diversos estudos mostrando associação positiva entre automedicação e doenças menos graves, muitas vezes de remissão espontânea ${ }^{(13,18)}$, assim como entre sinais e sintomas menores e de caráter mais agudo, como dor de cabeça e febre ${ }^{(20-21)}$. Relata-se, no presente estudo, que a maioria dos entrevistados que fez uso da prática de automedicação indicou o predomínio da cefaleia como sintoma desagradável e que, quando questionados se lembravam do nome do medicamento que usaram por conta própria, a dipirona foi a mais citada, assim como em resultados de outras pesquisas ${ }^{(12,23)}$.

Torna-se o uso de analgésicos pelos pacientes renais crônicos hemodialíticos de extrema importância para o controle das diversas situações de dor com as quais se deparam no dia a dia, pois a doença pode piorar a qualidade do sono e até facilitar uma piora física e funcional, e, até mesmo, desencadear transtornos depressivos ${ }^{(24)}$. Percebe-se, assim como em outros trabalhos ${ }^{(15,23)}$, que os antiinflamatórios não esteroidais (AINEs) também estiveram entre os 
medicamentos mais frequentemente usados sem receita. Esses fármacos deveriam ser evitados por pacientes que ainda possuem uma boa diurese residual ${ }^{(24)}$, pelo seu potencial considerável para interações medicamentosas e reações adversas e pelo aumento do risco cardiovascular ${ }^{(23)}$. Adverte-se que, apesar de a dipirona e alguns AINEs serem medicamentos isentos de prescrição, ou seja, de venda livre, ambos podem ser nocivos à saúde ${ }^{(18,19,23,25)}$, o que leva ao questionamento acerca do real benefício e promoção à saúde dessas práticas de autocuidado ou o cumprimento do tratamento em pacientes com doenças crônicas, em especial a DRC.

Verificou-se em todas as situações de uso de medicamento por conta própria, que não foi necessária a apresentação de receita no momento da compra, o que sugere o descumprimento das normas da legislação. Detalha-se que, apesar do risco de aumento de complicações diante de tal prática, a maioria dos entrevistados do atual estudo que se automedicaram disseram saber como usar o remédio e que melhoraram após o uso, o que, somado ao déficit das estruturas públicas de saúde insuficientes em atender à demanda de uma sociedade carente, torna a prática de automedicação uma opção de autocuidado, assim como relatado em outro estudo(15).

Expõe-se que, dos participantes que relataram ter abandonado por conta própria o uso de algum medicamento prescrito, a maioria alegou mal-estar quando o tomou, comunicando ao médico tal ação. Supõe-se que, quando o paciente age de tal maneira, suspendendo o medicamento que atribuiu ser a causa de um malefício, ele não costuma comunicar à equipe de saúde por medo de não ser compreendido ou de receber nova prescrição, de outro medicamento, e este provoque os mesmos efeitos colaterais ${ }^{(26)}$.

No presente estudo, apesar da maioria dos participantes possuir renda familiar que não ultrapassa dois salários mínimos, o gasto com medicamentos não pareceu ser algo preocupante, já que o preço do medicamento adquirido para se automedicar não influenciou na aquisição em $80 \%$ das situações. Corroborando esses dados, a literatura demonstra que a renda não é fator preponderante para a decisão de se automedicar ${ }^{(21)}$. Conhece-se, também, que a automedicação é uma alternativa de baixo custo e uma primeira opção de resposta frente à doença para as pessoas de baixo rendimento econômico ${ }^{(23)}$.

Observou-se associação estatística entre a automedicação e as variáveis escolaridade e uso de medicamentos guardados em casa na atual pesquisa. $\mathrm{E}$, dos pacientes que disseram ter feito uso de medicamentos guardados em casa, a porcentagem maior foi por meio de sobras de receitas anteriores, prática de relato comum também em outros estudos ${ }^{(13,19,23)}$. Essa prática pode ser um fator contribuinte em aumentar a resistência antimicrobiana e falhas de tratamento, assim como em piorar a qualidade dos medicamentos ${ }^{(21)}$, propiciando o uso de medicamentos vencidos inclusive. Ressalta-se que, no presente estudo, $73 \%$ relataram que costumam verificar a data de validade dos medicamentos.

Associa-se à variável escolaridade a prática de automedicação, sendo que, quanto maior o nível educacional, mais frequente é o ato de se automedicar ${ }^{(12)}$. Supostas explicações para isso seriam que o conhecimento, as habilidades e o acesso a informações tornam os pacientes mais confiantes para tomar a sua decisão na escolha pelo medicamento mesmo sem prescrição médica ${ }^{(27)}$.

Descreve-se que, apesar das dificuldades metodológicas encontradas (viés de informação e impossibilidade de validação das respostas dos entrevistados; viés de memória quanto ao uso de medicamentos; dificuldades envolvidas no conceito de automedicação), semelhantes às de outros estudos relacionados ao tema(18,20,23), os resultados alcançados na presente pesquisa são relevantes para a adoção de estratégias voltadas ao uso mais racional dos medicamentos, ao aprimoramento da assistência e segurança dessa população, principalmente considerando a carência de estudos envolvendo pacientes renais crônicos hemodialíticos. Informase que já existe relato, na literatura, de programas de manejo terapêutico dos medicamentos pelos farmacêuticos, reduzindo custos com medicamentos, as taxas e o tempo de hospitalizações, até mesmo a mortalidade ${ }^{(28)}$, em pacientes renais crônicos em diálise.

\section{CONCLUSÃO}

Há baixa frequência de automedicação na população de renais crônicos investigada, sendo associada a sua ocorrência ao uso de medicamentos guardados em casa, à baixa escolaridade, a problemas detectados como falha na comunicação, ao descumprimento de normas de comercialização e ao uso de sobras de medicamentos, que podem gerar falhas no tratamento e agravar comorbidades.

\section{CONFLITOS DE INTERESSE}

Os autores declaram que não existem conflitos de interesses. 


\section{CONTRIBUIÇÕES}

Lucas Brasileiro Lemos e Adriana Alves Nery contribuíram com a elaboração e delineamento do estudo; a aquisição, análise e interpretação de dados e a redação e/ou revisão do manuscrito. Gabriela Silva Moraes contribuiu com a elaboração e delineamento do estudo e com a redação e/ou revisão do manuscrito. Gisele da Silveira Lemos contribuiu com a aquisição, análise e interpretação de dados e com a redação e/ou revisão do manuscrito.

Artigo extraído da dissertação de mestrado intitulada: "Uso de medicamentos em pacientes renais crônicos hemodialíticos: características farmacoepidemiológicas". Universidade Estadual do Sudoeste da Bahia, 2015, 97 páginas.

\section{REFERÊNCIAS}

1. Ferraz FHRP, Rodrigues CIS, Gatto GC, Sá NM. Differences and inequalities in relation to access to renal replacement therapy in the BRICS countries. Ciênc Saúde Colet. 2017;22(7):2175-85.

2. Sesso RC, Lopes AA, Thomé FS, Lugon JR, Martins CT. Brazilian chronic dialysis survey 2016. J Bras Nefrol. 2017;39(3):261-6.

3. Oliveira JGR, Silva GB Jr, Vasconcelos JE Filho. Doença renal crônica: explorando novas estratégias de comunicação para promoção da saúde. Rev Bras Promoç Saúde. 2018;31(4):1-8.

4. Ministério da Saúde (BR), Secretaria de Vigilância em Saúde. Política Nacional de Promoção da Saúde. $3^{a}$ ed. Brasília: Ministério da Saúde; 2010.

5. Saran R, Robinson B, Abbott KC, Bragg-Gresham J, Chen X, Gipson D, et al. US Renal Data System 2019 Annual Data Report: epidemiology of kidney disease in the United States. Am J Kidney Dis. 2020;75(suppl 1).

6. Teixeira FIR, Lopes MLH, Silva GAS, Santos RF. Survival of hemodialysis patients at a university hospital. J Bras Nefrol. 2015;37(1):64-71.

7. Weir MR, Fink JC. Safety of medical therapy in patients with chronic kidney disease and end-stage renal disease. Curr Opin Nephrol Hypertens. 2014;23(3):306-13.

8. Gerlack LF, Werlang MC, Bós AJG. Drug related problems in outpatient older adults assisted in an university hospital in Rio Grande do Sul, Brazil. Rev Bras Farm Hosp Serv. 2015;6(1):13-7.

9. Secoli SR, Marquesini EA, Fabretti SC, Corona LP, Romano-Lieber NS. Self-medication practice trend among the Brazilian elderly between 2006 and 2010: SABE Study. Rev Bras Epidemiol. 2018;21(Suppl 2):E180007.

10. Pereira FGF, Araújo MJP, Pereira CR, Nascimento DS, Galiza FT, Benício CDAV. Automedication in active elderly people. J Nurs UFPE. 2017;11(12):4919-28.

11. Bertoldi AD, Pizzol TSD, Ramos LR, Mengue SS, Luiza VL, Tavares NUL, et al. Sociodemographic profile of medicines users in Brazil: results from the 2014 PNAUM survey. Rev Saúde Pública. 2016;50(Suppl 2):5s.

12. Prado MAMB, Franscisco PMSB, Bastos TF, Barros MBA. Use of prescription drugs and self-medication among men. Rev Bras Epidemiol. 2016;19(3):596-608.

13. Koley M, Saha S, Ghosh A, Ganguly S, Arya JS, Choubey G. Self-medication tendencies of patients visiting outpatient departments of Government homeophatic medical colleges and hospitals in West Bengal, India. Int J High Dilution Res. 2013;12(45):178-89.

14. Nepomuceno FCL, Melo IM Jr, Silva EA, Lucena KDT. Religiosidade e qualidade de vida de pacientes com insuficiência renal crônica em hemodiálise. Saúde Debate. 2014;38(100):119-28.

15. Machado-Alba JE, Echeverri-Cataño LF, Londoño-Builes MJ, Moreno-Gutiérrez PA, Ochoa-Orozco SA, RuizVilla JO. Social, cultural and economic factors associated with self-medication. Biomédica. 2014;34(4):580-8.

16. Domingues PHF, Galvão TF, Andrade KRC, Araújo PC, Silva MT, Pereira MG. Prevalence and associated factors of self-medication in adults living in the Federal District, Brazil: a cross-sectional, population-based study. Epidemiol Serv Saúde. 2017;26(2):319-30.

17. World Health Organization. Guidelines for ATC classification and DDD assignment [Internet]. Oslo: WHO 
Collaborating Centre for Drug Statistics Methodology; 2018 [acesso em 2019 Abr 12]. Disponível em: https:// www.whocc.no/atc_ddd_index_and_guidelines/guidelines/

18. Sawalha AF. A descriptive study of self-medication practices among Palestinian medical and nonmedical university students. Res Soc Adm Pharm. 2008;4(2):164-72.

19. Auta A, Banwat SB, Sariem CN, Shalkur D, Nasara B, Atuluku MO. Medicines in Pharmacy Students' Residence and Self-medication Practices. J Young Pharm. 2012;4(2):119-23.

20. Barros ARR, Griep RH, Rotenberg L. Self-medication among nursing workers from public hospitals. Rev Latino-Am Enfermagem. 2009;17(6):1015-22.

21. Galato D, Madalena J, Pereira GB. Self-medication among university students: the influence of the field of study. Ciênc Saúde Colet. 2012;17(12):3323-30.

22. Institute of Medicine. The healthcare imperative: lowering costs and improving outcomes: workshop series summary [Internet]. Washington, DC: The National Academies Press; 2010 [acesso em 2019 Abr 12]. Disponível em: http://books.nap.edu/catalog.php?record_id=12750

23. Suleman S, Ketsela A, Mekonnen Z. Assessemnt of self-medication practices in Assendabo town, Jimma zone, southwestern Ethiopia. Res Soc Adm Pharm. 2009;5(1):76-81.

24. Baldoni AO, Ayres LR, Martinez EZ, Dewulf NLS, Santos V, Obreli-Neto PR, et al. Pharmacoepidemiological profile and polypharmacy indicators in elderly outpacients. Braz J Pharm Sci. 2013;49(3):443-52.

25. Agência Nacional de Vigilância Sanitária (BR). Resolução da Diretoria Colegiada (RDC) $n^{\circ} 98$, de $1^{\circ}$ de agosto de 2016. Brasília: Anvisa; 2016.

26. Santoro D, Satta E, Messina S, Costantino G, Savica V, Bellinghieri G. Pain in end-stage renal disease: a frequent and neglected clinical problem. Clin Nephrol. 2013;79(Suppl 1):2-11.

27. Jain D, Green JA. Health literacy in kidney disease: review of the literature and implications for clinical practice. World J Nephrol. 2016;5(2):147-51.

28. St Peter WL, Wazny LD, Patel UD. New models of chronic kidney disease care including pharmacists: improving medication reconciliation and medication management. Curr Opin Nephrol Hypertens [Internet]. 2013 [acesso em 2019 Abr 12];22(6):656-62. doi: 10.1097/MNH.0b013e328365b364

\author{
Endereço para correspondência: \\ Lucas Brasileiro Lemos \\ Universidade Estadual do Sudoeste da Bahia - UESB \\ Departamento de Saúde II \\ Rua José Moreira Sobrinho, s/n \\ Bairro: Jequiezinho \\ CEP: 45200-000 - Jequié - BA - Brasil \\ E-mail: brasileirolemos@gmail.com
}

Como citar: Lemos LB, Moraes GS, Lemos GS, Nery AA. Automedicação em pacientes renais crônicos hemodialíticos. Rev Bras Promoç Saúde. 2020;33:9906. 